\title{
Attenuation of Oscillations in a Mechanical System Using a Vibrational Parameter
}

\author{
J. Tellez \\ Control Department \\ Cinvestav IPN - UPVM \\ Mexico City, Mexico \\ jtellez@ctrl.cinvestav.mx
}

\author{
J. Collado \\ Control Department \\ Cinvestav IPN \\ Mexico City, Mexico \\ jcollado@ctrl.cinvestav.mx
}

\begin{abstract}
In this work we attenuate the oscillations of a mechanical system using a vibrational control term introduced in the elasticity of a spring. First we must analyze the stability of the system due to the induction of periodic excitation can cause parametric resonance.
\end{abstract}

Keywords-Attenuation, Stability analysis, Parametric excitation, Vibration.

\section{INTRODUCTION}

The linear differential equations with periodic coefficients have been studied extensively motivated by applications in the fields of physics and engineering [1], for example in the study of suspension bridges [2], rotating discs, elastic pendulums, the Kapitza pendulum [11], vibrations in rotating shafts [10], propagation of acoustic or electromagnetic waves or a system of a crane mounted on a ship under the action of the waves to name just a few.

Basis of such equations were established by several authors including Floquet [8], Hill [9], Mathieu [7], Faraday [6], and others. Very detailed and deep presentations are the works of Yakubovich and Starzhinskii [2] and [12].

In this work we analyze the stability of a mechanical double harmonic oscillator subject to a parametric periodic excitation induced in the elasticity of a spring and we increase the attenuation of the system temporal response adding a vibrational parameter, we characterize the values of the parameter in order to achieve the maximum attenuation.

II.

PERIODIC SySTEMS

The periodic systems are a kind of systems described by the equation:

$$
\dot{x}=A(t) x \quad x \in \mathbb{R}^{n}, \quad-\infty<t<\infty
$$

Where the elements of $\mathrm{A}(\mathrm{t})$ are piecewise continuous periodic functions, with minimum period $\mathrm{T}$.

$$
A(t)=A(t+T)
$$

\section{A. Properties of periodic systems}

The solution of (1) is:

$$
x(t)=\phi(t, t o) x(t o)
$$

In general $\phi(t, t o)$ cannot be computed for the periodic case, except for some very specific systems.

Theorem 1: [3],[15]

A system like (1), with $A(t)=A(t+T)$ has at least one no identically zero solution, such that

$$
x(t+T)=\lambda x(t), \quad \forall t, \quad \lambda \neq 0
$$

Let a homogeneous system like (1), then there exist a periodic and invertible matrix $P(t)=p(t+T) \in \mathbb{R}^{n x n}$ and a constant matrix $R \in \mathbb{R}^{n x n}$ such that (See [1] and [2]).

$$
\phi\left(t_{0}+T\right)=P^{-1}\left(t_{0}\right) e^{R T} P\left(t_{0}\right)
$$

Last equation shows that the monodromy matrix is similar to $e^{R T}$.

If we assume $t_{0}=0$, then for any $t \geq 0$, t can be written as: $t=k T+\tau, k \in \mathbb{Z}^{+}$(non negative integers) $\tau \in[0, T)$.

$$
\begin{gathered}
\mathrm{x}(\mathrm{t})=\phi(\mathrm{t}, 0) \mathrm{x}(0) \\
=\phi^{k}(\mathrm{~T}+\tau, \tau) \phi(\tau, 0) \mathrm{x}(0)=\mathrm{M}^{k} \phi(\tau, 0) x(0)
\end{gathered}
$$

Boundedness of $x(t)$ depends only on the boundedness of the factor $\mathrm{M}^{k}$ due to $\phi(\tau, 0)$ is bounded because $\tau \in[0, T)$ and a linear System cannot go to infinity in finite time; and $\mathrm{x}(0)$ is also bounded.

The above result show that the system stability depends only of the eigenvalues of the monodromy matrix.

\section{B. The Hill's equation}

The Hill's equation is a second order linear homogeneous equation with real periodic coefficients [4], without loss of generally $\mathrm{p}(\mathrm{t})$ is a zero average function.

$$
\begin{gathered}
\ddot{y}+(\alpha+\beta p(t)) y=0 \\
p(t+T)=p(t), \int_{0}^{T} p(t) d t=0
\end{gathered}
$$

Its stability analysis is much more involved than linear differential equation with constant coefficients [5],[2].

Hill's equation (2) can be represented in state space form:

$$
\dot{x}=\left[\begin{array}{cc}
0 & 1 \\
-\alpha-\beta p(t) & 0
\end{array}\right] x
$$

where $x=\left[\begin{array}{ll}y & \dot{y}\end{array}\right]^{T}$ 


\section{Stability of Hill's Equation}

For linear systems described by ordinary differential equa- tion with periodic coefficients, the Floquet's theory can be used to reduce the problem of stability to the calculus of eigenvalues of the Monodromy Matrix, which is roughly speaking, the state transition Matrix evaluated after one Period.

\section{Monodromy matrix}

The Monodromy matrix is defined in [3] as:

$$
M:=\phi\left(t_{0}+T, t_{0}\right)
$$

where $\phi\left(t, t_{0}\right)$ is a fundamental matrix of the system $\dot{\mathrm{x}}=\mathrm{Ax}$ and $\mathrm{T}$ is the minimum period of $\mathrm{p}(\mathrm{t})$.

It may be proved that $\sigma(M)$ does not depends on $t_{0}$ [3]; where $\sigma(\mathrm{R})$ represents the set of eigenvalues including multiplicity of the square matrix R.

\section{E. Stability criteria for the Hill's equation}

Theorem 2: The periodic system (3) is (See [4]):

Asymptotically stable if and only if:

Stable if:

$$
\sigma(M) \subset \stackrel{\circ}{D_{1}}:=\{z \in \mathbb{C}:|z|<1\}
$$

$$
\sigma(\mathrm{M}) \subset \mathrm{D}_{1}:=\{\mathrm{z} \in \mathbb{C}:|\mathrm{z}| \leq 1\}
$$

and $\lambda_{\mathrm{i}} \in \sigma(M):\left|\lambda_{\mathrm{i}}\right|=1$, is a simple root of its minimal polynomial $\mathrm{m}_{\mathrm{M}}(\mathrm{t})$.

Unstable if and only if $\exists \lambda_{\mathrm{i}} \in \sigma(\mathrm{M}):\left|\lambda_{\mathrm{i}}\right|>1$, or

$\sigma(M) \subset D_{1}$ is a multiple root of $m_{M}(t)$.

where $\mathrm{M}$ is any monodromy matrix of system (3). 1

This theorem gives us necessary and sufficient conditions about the system stability using the eigenvalues of the monodromy matrix [4].

\section{F. Vibrational control}

Given the system described by the equation

$$
\dot{x}=A x, x \in E_{n}, A=\left\|a_{i j}\right\|_{i, j=1}^{n}, a_{i j}=\text { constant }
$$

If we vibrate the elements of the matrix A with the control law $B(t)=\left\|b_{i j}\right\|_{i, j=1}^{n}$, where $b_{i j}=\sum_{s=1}^{\infty} a_{i j}^{s} \sin \left(s \varpi_{i j} t+\right.$ $\left.\theta_{\mathrm{ij}}^{\mathrm{S}}\right)$ are periodic functions with zero mean value, then:

$$
\dot{\mathrm{x}}=[\mathrm{A}+\mathrm{B}(\mathrm{t})] \mathrm{x}
$$

Vibrations used for control and stabilization can be arbitrary periodic functions in form and frequency on the understanding that they must have zero mean value [14].

Definition 3: The system described by the equation (5), will be said vibrationally stabilizable, if there exists a periodic matrix $\mathrm{B}(\mathrm{t})$ (with zero mean value), such that the trivial solution $x=0$ of the system (6) is asymptotically stable [14].

1 Any monodromy matrix means that does not matter for which value of t0 is calculated.

\section{DEVELOPMENT}

Now, using the mathematical model of the double harmonic oscillator, we make a stability analysis when one of its parameters varies periodically.

\section{A. The double harmonic oscillator}

The diagram of the double harmonic oscillator is:

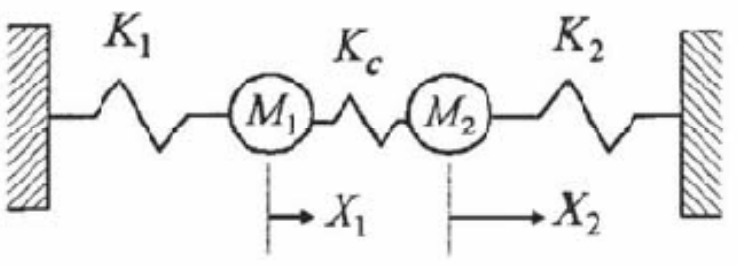

Figure 1. Double harmonic oscillator scheme.

The movement equations of the system are (See [13]):

$$
\begin{aligned}
& m_{1} \frac{d^{2} x_{1}}{d t^{2}}+\left(k_{1}+k_{c}\right) x_{1}-k_{c} x_{2}=0 \\
& m_{2} \frac{d^{2} x_{2}}{d t^{2}}+\left(k_{2}+k_{c}\right) x_{2}-k_{c} x_{1}=0
\end{aligned}
$$

The matrix representation of the system is:

$$
\ddot{x}+\left[\begin{array}{cc}
\frac{k_{1}+k_{c}}{m_{1}} & \frac{-k_{c}}{m_{1}} \\
\frac{-k_{c}}{m_{2}} & \frac{k_{2}+k_{c}}{m_{2}}
\end{array}\right] x=0
$$

Factorizing each spring term and consider a viscous friction term (air friction), the system equations looks like:

$$
\begin{aligned}
& \ddot{x}+\left[\begin{array}{ll}
\gamma & 0 \\
0 & \gamma
\end{array}\right] \dot{x}+\left\{\left[\begin{array}{cc}
\frac{1}{m_{1}} & -\frac{1}{m_{1}} \\
-\frac{1}{m_{2}} & \frac{1}{m_{2}}
\end{array}\right] k_{c}+\right. \\
& \left.+\left[\begin{array}{cc}
\frac{1}{m_{1}} & 0 \\
0 & 0
\end{array}\right] k_{1}+\left[\begin{array}{cc}
0 & \frac{1}{m_{2}}
\end{array}\right] k_{2}\right\} x=0
\end{aligned}
$$

In the equation (7), if the stiffness of any spring varies periodically, the equation (7) is a Hill's type matrix equation.

\section{B. Temporal response of the system}

The temporal response of the equation (7) considering $k 1=1, k 2=1, k c=1, \gamma=0.0005, m 1=1, m 2=1, w$ $=1$

calculated over Matlab Simulink is:

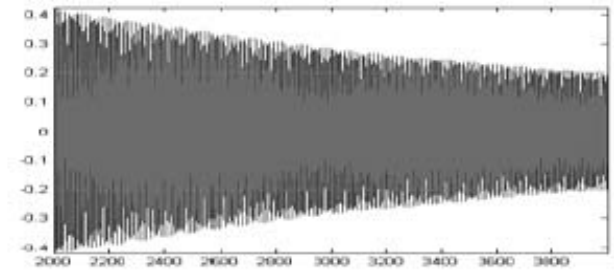

Figure 2. Free temporal response of the system, considering a damping term $\gamma=0.0005$ an initial displacement condition $x_{1}(0)=1$. 
Observe that the value at $4000 \mathrm{sec}$. has an amplitude about 0.22 due to the damping term of the equation (7).

We want to increase the damping of the system using a vibrational control term and attenuate the oscillations.

\section{Stability analysis of the system in presence of parametric excitation}

We must ensure the stability of the system when we add the periodic excitation term, for this purpose, we analyze the equation (7) and considering that the stiffness of the spring where

$\square \mathfrak{1}$ varies periodically in time, as $k_{c}=\alpha+\beta \cos (w t)$,

$k_{2}=1, k_{c}=1, \gamma=0, m_{1}=1, m_{2}=1, w=1$.

The system equation is:

$$
\ddot{x}+\left\{\left[\begin{array}{ll}
1 & 0 \\
0 & 1
\end{array}\right]+(\alpha+\beta \cos (\varpi t))\left[\begin{array}{cc}
1 & -1 \\
-1 & 1
\end{array}\right]\right\} x=0
$$

Using a Matlab algorithm that draws the stability diagram of figure 3 .

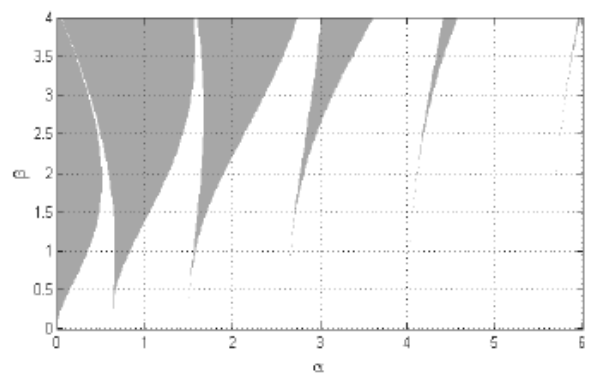

Figure 3. Stability diagram of the equation (8) considering a periodic excitation over the stiffness of $k c$.

The algorithm that draw the stability diagram works as follows. First, we calculate the System Monodromy matrix column by column, for that we solve numerically de equation (8) one time for each vector of initial condition defined by each one of the identity matrix columns, next that we obtain the eigenvalues of the monodromy matrix and evaluate them in the stability criterion given in the theorem 2 . We draw a gray point if the system is unstable, white points are the stable zones (See [16]). The tongues consist of unstable areas are the so called Arnold Tongues [4].

\section{Inducing damping of the double harmonic oscillator}

The goal of this work is to reduce the oscillation's amplitude of the system (increase damping), for this purpose we incorporate a periodic parametric oscillation over the stiffness of the $k c$ spring of the form:

$$
k_{c}=\alpha+\beta \cos (w t)
$$

We need to chose the values of $\alpha, \beta$ and that reduced the oscillation and ensure stability of the system.

To achieve this, in the previous section we construct the stability diagram of the system, considering a periodic variation of $k c$. .(See figure 3 ).

In order to appreciate the effects of parametric excitation induced in . we leave one of the excitations parameters fixed and vary only the remaining two.
1) Case 1: Using a fixed $\omega$ : Given $\omega=1$ we chose the values of $\alpha$ and $\beta$. to increase the damping term in the equation (7), for example we chose $\beta=1.5$, and adjust the $\alpha$ value to get the best possible vibrational control term for attenuate the oscillations.

The $\alpha$ value is chosen in the stable region located in the middle of the two Arnold tongues (See figure 4).

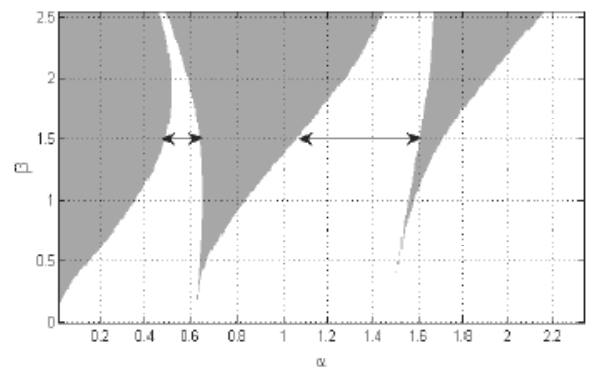

Figure 4. Stable region between two Arnold tongues.

The induced damping for the vibrational control term $k c=\alpha+\cos (t)$ is greater for the minimum value of $\alpha=1.4$ in the middle of the stable interval, and decrease in the other values of this parameter.

In the figure 5 the attenuation of the system is about 30\% greater that the system without the parametric excitation, the system amplitude response is 0.152 (in the original system is $0.22)$.

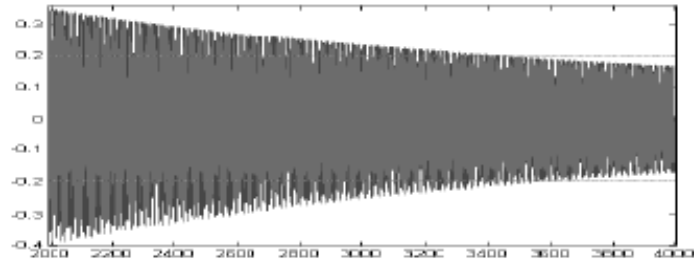

Figure 5. Temporal response for the system considering $k c=1.4+1.5 \cos (t)$, this excitation term provides greater attenuation of oscillations in this range

If $\alpha$ is greater of this value the induced damping is not increase. This is, for a given values of $\beta$ and $\omega$ there exists a minimum value of $\alpha$ that give us the maximum induced damping. This can be seen in the Figure 6.

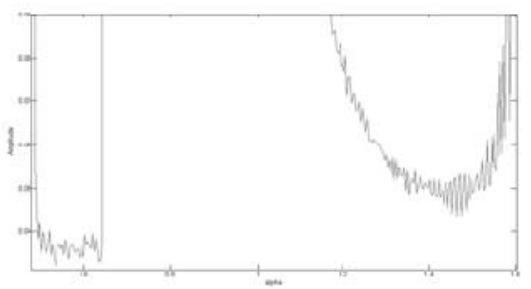

Figure 6 . The figure shows the attenuation pattern in the first and second stability intervals using $\beta=1.5, w=1$. We can see that the maximum induced attenuation is achieved in the first stability interval. 
Now we change the value of $\beta$ to 0.5 and repeat the experiment, we try to determine the $\alpha$ value to reach the maximum parametric attenuation.

In the next table we show the values of the excitation parameter $\alpha$ to obtain the maximum attenuation to different values of $\beta$.

\begin{tabular}{|l|l|l|l|}
\hline$w$ & $\beta$ & $\alpha$ & amplitude \\
\hline 1 & 0.5 & $\mathbf{1 . 6}$ & 0.152 \\
\hline 1 & 1.5 & $\mathbf{1 . 4}$ & 0.152 \\
\hline 1 & 2.5 & 0.49 & 0.152 \\
\hline
\end{tabular}

In the three examples of this case, we can see that for a greater $\beta$ the $\alpha$ value to reach the maximum attenuation is smaller.

2) Case 2: Using a fixed $\alpha$ : In this case we start with the first example of the previous case. Given fixed $\alpha=1.4$ and selecting $\beta=1.5$.

We note when the frequency is increase the maximum attenuation is reached for a smaller $\alpha$.Using a reduced $\alpha=1$ we adjust the frequency parameter to ensure the best possible incorporated damping term. The periodic excitation term has the form $k_{c}=1+1.5 \cos (w t)$.

For $=5$ we achieve the maximum induced damping due to the periodic excitation term (temporal response amplitude is 0.152 ).

The system response is:

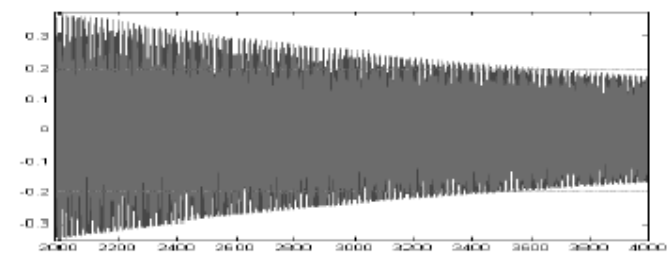

Figure 9. Temporal response of the system using $k c=1+$

$1.5 \cos (5 t)$. This figure shows that it is possible to induce damping varying the frequency of the periodic excitation too.

In the frequency variation case from the maximum attenuation, the reduction of the frequency cause a smaller attenuation in the system response, but in the same way the increase of the frequency has the same effect.

\section{E. Conclusion}

We report here an example of a relatively simple mechanical system, in this case a double harmonic oscillator, we can see that if there is periodic variation in some of its parameters, the damping of the system increase if the values of the parameters are adequate. It is possible to reach the same final amplitude in the system response approximately in three quarter part of time, and it's possible to attenuating the final amplitude approximately $30 \%$. In addition for a greater $\beta$ in the parametric excitation the maximum induced attenuation is reached for a smaller value of $\alpha$ and if the frequency is increase the value of $\alpha$ for a fixed $\beta$ in smaller too.

It is possible too, add the parametric vibrational control in more than one of the parameters of the system.

\section{REFERENCES}

[1] V.A. Yakubovich and V.M. Starzhinskii. Linear differential equations with periodic coefficients. Vol. 1. John Wiley, Jerusalem, 1975. Ch. 4.

[2] V.A. Yakubovich and V.M. Starzhinskii. Linear differential equations with periodic coefficients. Vol. 2. John Wiley, Jerusalem, 1975.

[3] D.W. Jordan and P. Smith. Nonlinear Ordinary Differential Equations. Oxford University Press. USA 2007. 4th Edition.

[4] W. Magnus and S. Winkler. Hill's Equation. Dover. USA 2004.

[5] V. Bolotin. The dynamic stability of elastic systems. Holden Day Inc. USA 1964.

[6] M. Faraday. On a peculiar class of acoustical figures and on certain forms assumed by a group of particles upon vibrating elastic surfaces. Transactions Royal Society (1831), pp. 299-318.

[7] E. Mathieu. Memoire sur le mouvement vibratoire d'une membrane de forme elliptique. J. Math Pures Appl. 13 (1868), pp. 137-203.

[8] G. Floquet. Sur les équations différentielles linéaires à coefficients périodiques. Annales de la Ecole Normal Superior (1883), pp.47-89.

[9] W.G. Hill. On the part of the motion of the lunar perigee. Acta Math 8 (1886). pp. 1-36.

[10] G. Genta. Vibrations dynamics and control. Springer USA 2009.

[11] A.A. Seyranian, et al. The stability of an inverted pendulum with a vibrating suspension point. Journal of Applied Mathematics and Mechanics, Volume 70, Issue 5, Elsevier. Rusia 2006, pp.754-761.

[12] V.A. Yakubovich and V.M. Starzhinskii. Parametric Resonance in Linear Systems, Nauka, 1982 (In Russian), pp. 209-212.

[13] F.Axisa. Modelling of Mechanical Systems. Discrete Systems Volume 1. Kogan Page Science. USA 2004.

[14] S. Meerkov. Vibrational Control Theory. Moscow Institute of Science. USSR.

[15] L. Cesari. Asymptotic behavior and stability problems in ordinary differential equations, 2nd ed. Springer-Verlag, 1963.

[16] H. Jardón. Tésis: Control de sistemas modelados por la ecuación de Hill con base en discretización. DCA CINVESTAV IPN, México, D.F. 2010 . 